\title{
ANN based soft sensor model for reactive distillation column
}

\author{
Gaurav Kataria*, Kailash Singh and Rajeev Kumar Dohare \\ Department of Chemical Engineering, Malaviya National Institute of Technology, Jaipur, India
}

\section{(C2016 ACCENTS}

\begin{abstract}
To cope up with the strict emission and product quality standards the process industries are stern for the maintenance of the product quality and waste emission. For process industries, online hardware analyzers are costly alternate for analyzing the product quality, they are switching to online soft sensors which are cheap and efficient in use. In this work, soft sensor based on artificial neural network (ANN) has been developed. The motive of the sensor is to measure the immeasurable primary variables of the reactive distillation column i.e. product concentration, using the data of easily measurable secondary variables i.e. tray temperatures. ANN soft sensor has been trained using Levenberg-Marquardt algorithm. The predictions of the soft sensor are compared with the MATLAB mathematical model outputs.
\end{abstract}

\section{Keywords}

Soft sensor, ANN, Reactive distillation column, Levenberg-marquardt algorithm.

\section{Introduction}

In chemical industries quality standards and emission standards are getting strict. To meet the market and pollution control board demands, online monitoring of quality variables is essential for an industry. As concentration is a direct measure of product quality, so it is better to monitor the concentration of product in real time. The on-line measurement of concentration is not an easy and cheap task. Hardware estimators are costlier and also produce some time-lag. So, Soft sensors are suggested to measure the quality variable or concentration online. using easily measured variables such as tray temperatures, reboiler duty, etc.. Reactive distillation is a process that carries reaction and separation processes in a single still. Reversible reactions are operated in Reactive distillation column (RDC) so that equilibrium can be restricted in the process. The prime motive of RDC is to separate the products of the reaction so that Le-Chatelier's principle can restrain the backward reaction. It leads to better selectivity, better conversion, avoidance of Azeotropes formation and better heat utilization in the reactive distillation column. Esterification reaction is a reversible reaction which has been studied extensively in the RDC's. Esterification involves the formation of an ester from the reaction of carboxylic acid and alcohol in the presence of an acidic catalyst.

*Author for correspondence

182
For maintaining the performance of an RDC, On-line monitoring of the product concentration in crucial for RDC's. Soft sensors can be developed using linear models or non-linear models. Linear model includes principle component analysis (PCA) [1-6] and partial least square (PLS) [2-8]. While, non-linear method includes artificial neural network (ANN) [7-15], and support vector regression (SVR) [5, 16-18]. Whereas, [19] and [20] proposed the first soft sensors for online monitoring in reactive distillation column's (RDC). They used extended Kalman Filter approach to estimate the product purity in the column.

An artificial neural network is an interesting approach for handling non-linearity in the process model. In this paper ANN has been used to estimate the product concentration for the reactive distillation column. ANN network is based on LevenbergMarquardt algorithm for optimization of the network values.

\section{Reactive distillation column model}

The mathematical model for reactive distillation was developed using MATLAB Software. Butyl Acetate esterification was studied for the same. The reaction taking place in the column can be shown as:

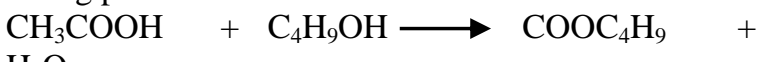
$\mathrm{H}_{2} \mathrm{O}$

Acetic Acid n-Butanol n-Butyl Acetate Water Assumptions taken for the mathematical model are:

1. The reaction is proceeding only in the liquid phase 
2. Reaction is occurring only in the Reactive Section

3. Vapor holdup is assumed to be negligible

4. System is obeying UNIQUAC thermodynamic

5. Phase splitting is considered only in the decanter mode

6. Constant tray pressure drop is considered.

The stages are numbered from top to the bottom. Where, when $1^{\text {st }}$ tray is condenser and $\mathrm{NT}^{\text {th }}$ tray is reboiler.

Modeling equation [20] and [21] for the esterification reaction in reactive distillation column can be shown as:

Material Balance on $\mathrm{i}^{\text {th }}$ tray

$\frac{d M_{i}}{d t}=L_{i-1}+V_{i+1}-L_{i}-V_{i}+F_{i}$

Component Material Balance on $i^{\text {th }}$ tray and $j^{\text {th }}$ element

$\frac{d M_{i} x_{i, j}}{d t}=L_{i-1, j} x_{i-1, j}+V_{i+1} y_{i+1, j}-L_{i} x_{i, j}-V_{i} y_{i, j}+$

$F_{i} x_{f i, j}+\Delta R_{i}$

Enthalpy Balance on ith tray

$\frac{d M_{i} h_{i}}{d t}=L_{i-1} h_{i-1}+V_{i+1} H_{i+1}-L_{i} h_{i}-V_{i} H_{i}+F_{i} h_{f i}$

Material Balance on Condenser ( $1^{\text {st }}$ Tray)

$\frac{d M_{1}}{d t}=V_{2}-R-D$

Component Material Balance on Condenser

$\frac{d M_{1} x_{1, j}}{d t}=V_{2} y_{2, j}-(R+D) x_{1, j}$

Enthalpy Balance on Condenser

$\frac{d M_{1} h_{1}}{d t}=V_{2} H_{2}-(R+D) h_{1}-Q_{C}$

Material Balance on Reboiler ( $\mathrm{NT}^{\text {th }}$ tray)

$\frac{d M_{N T}}{d t}=L_{N T-1}-V_{N T}-B$

Component Material Balance on Reboiler

$\frac{d M_{N T} x_{N T, j}}{d t}=L_{N T-1, j} x_{N T-1, j}-V_{N T} y_{N T, j}-B x_{N T, j}$

Enthalpy Balance on Reboiler

$\frac{d M_{N T} h_{N T}}{d t}=L_{N T-1} h_{N T-1}-V_{N T+1} H_{N T+1}-B h_{N T}+$

$Q_{B}$

The above mathematical model has been solved in MATLAB software for the collection of temperature and concentration data which is further used as input data for the soft sensor.

\section{ANN soft sensor}

Artificial neural network (ANN) is a reliable tool for dealing with complex nonlinear models. ANN architecture comprises of an input layer, hidden layer, and an output layer. Hidden layers contain various neurons which pass information through a weighted signal from one neuron to other, and this continues until the output is obtained. The performance of the network can be regulated by adjusting the weights between the neurons.

The concentration of n-Butyl Acetate for a reactive distillation column has been estimated by a 3-layered artificial neural network. ANN based soft sensor used tray temperatures as input variables. The network was trained using Levenberg-Marquardt optimization technique. LM technique is opted as it can handle a complex model with a large number of parameters. LM also holds benefits of fast convergence as GaussNewton method and assured convergence as of steeper descent method.

The general model equation of a 3 layered Artificial Neural network can be shown as:

$$
\begin{aligned}
& y=f^{3}\left(L W^{3} f^{2}\left(L W^{2} f^{1}\left(I W^{1} x+b^{1}\right)+b^{2}\right)+\right. \\
& \left.b^{3}\right)
\end{aligned}
$$

Where,

$I W^{1}=$ Input Weight at Layer 1

$L W^{2}, L W^{3}=$ Layer Weight at Layer $2 \& 3$

$b^{1}, b^{2}, b^{3}=$ bias values at Layer $1,2 \& 3$

$x=$ Input Vector

$y=$ Output Vector

In this paper, input data for the soft sensor was obtained from MATLAB model of the reactive distillation column. Input data contain tray temperature data and n-Butyl Acetate concentration data at different reboiler duties. ANN developed using $80 \%$ of data for training the network, $10 \%$ for cross-validation of the network and rest $10 \%$ for testing the network [22]. Network was developed using 3 layer models with one input layer, one hidden layer and one output layer. Input layer contain 15 tray temperature variables, Hidden layer contain 5 neurons and output layer contain one output variable of n-Butyl Acetate mole fraction.

\section{Results}

An ANN based soft sensor was developed for estimation of product purity in RDC and results were obtained and compared with actual values. Figure 1 shows the perfect fit of the training data when the network has been trained with the total of 55 set of tray temperature as input variables and 55 set mole fraction as target variables. After training the network, the network was validated with the crossvalidation data as shown in Figure 2. It can be seen that the network has been cross-validated with MSE of $5.65 \times 10^{-6}$ and $\mathrm{R}^{2}$ value of 0.963 and network can be used for the testing purpose. In last, the network 
Gaurav Kataria et al.

has been tested with different sets of tray temperature with a little MSE value of $4.12 \times 10^{-6}$. data as shown in Figure 3. Testing data fitted finely

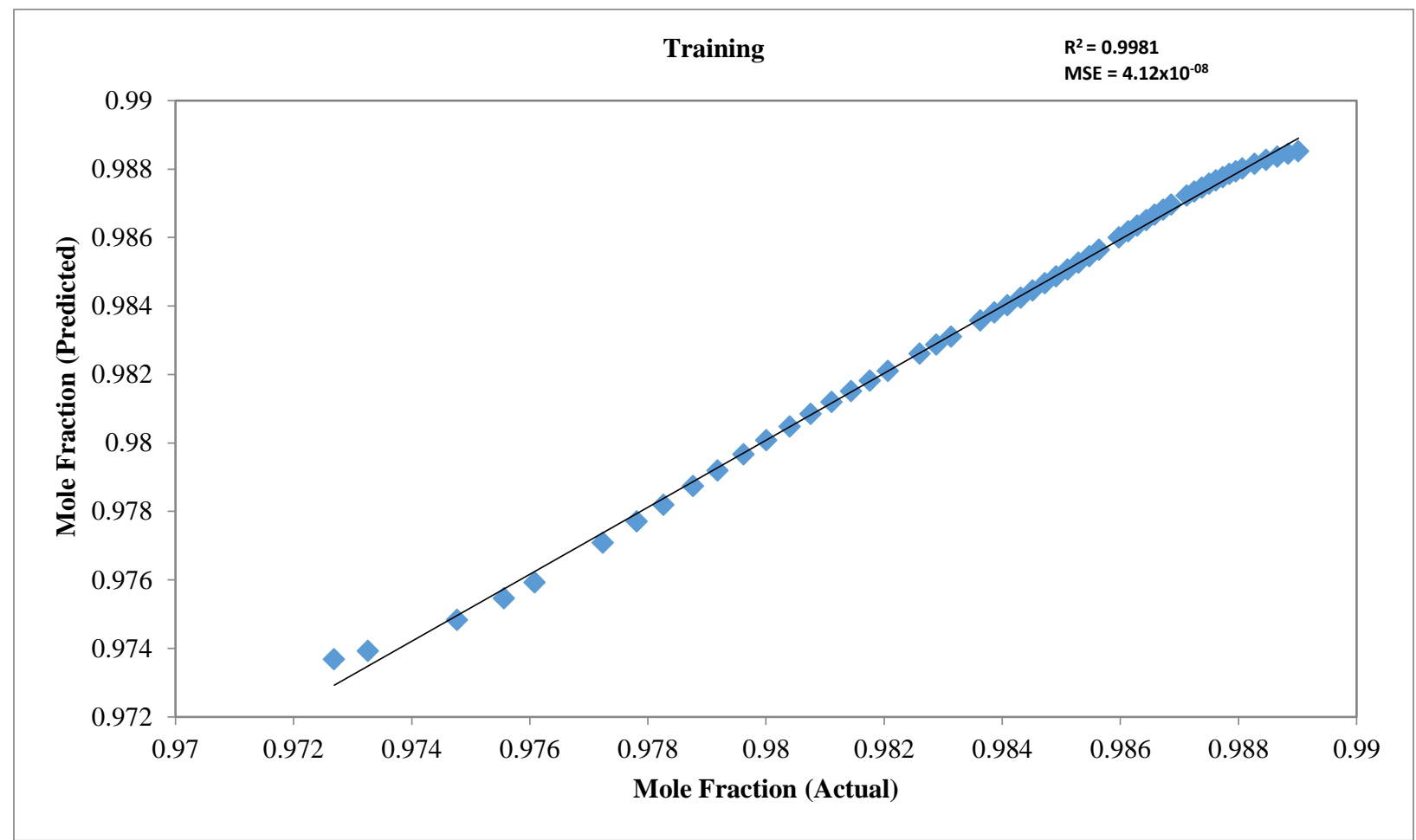

Figure 1 Training curve of network

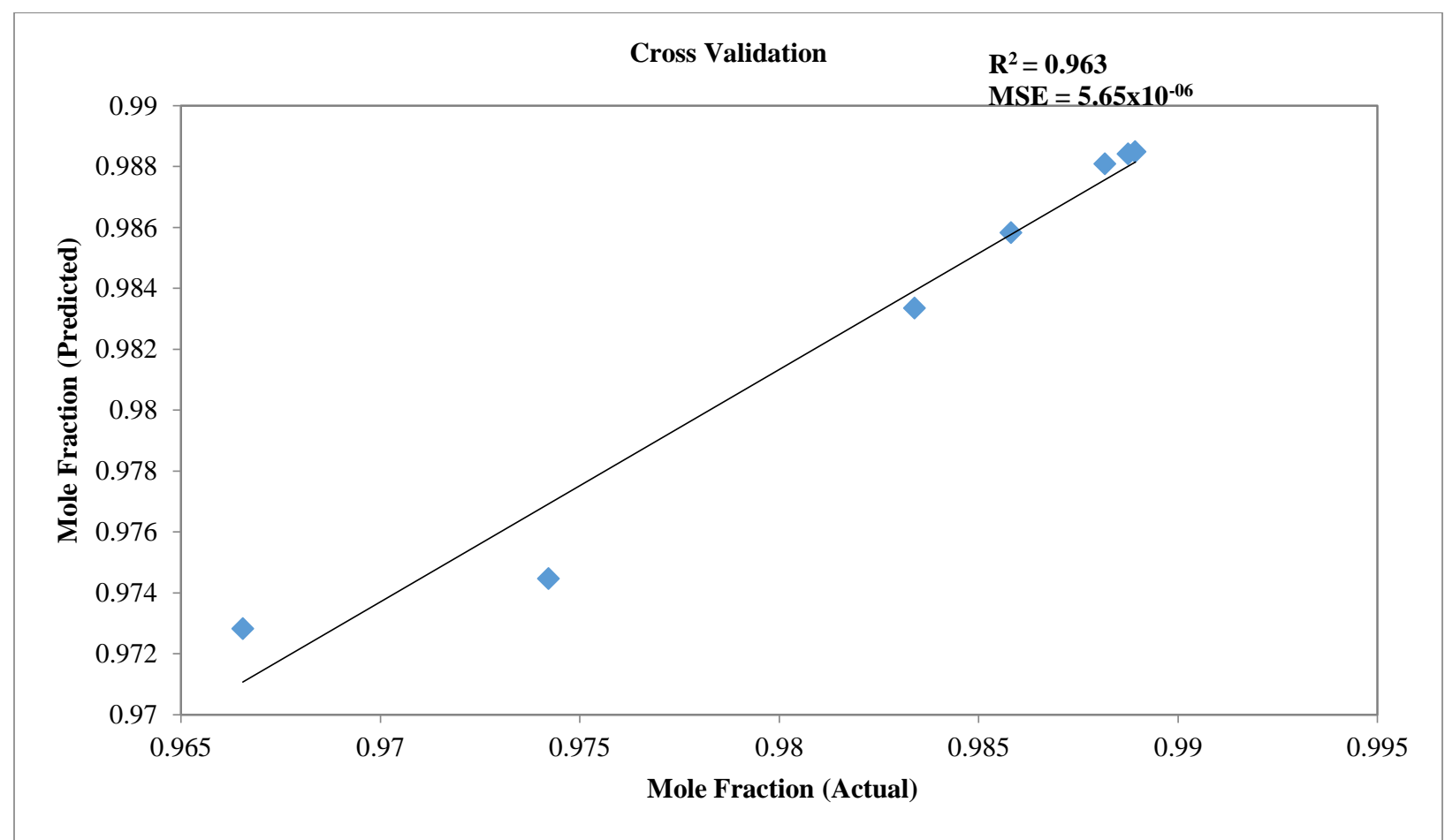

Figure 2 Cross validation curve of network 


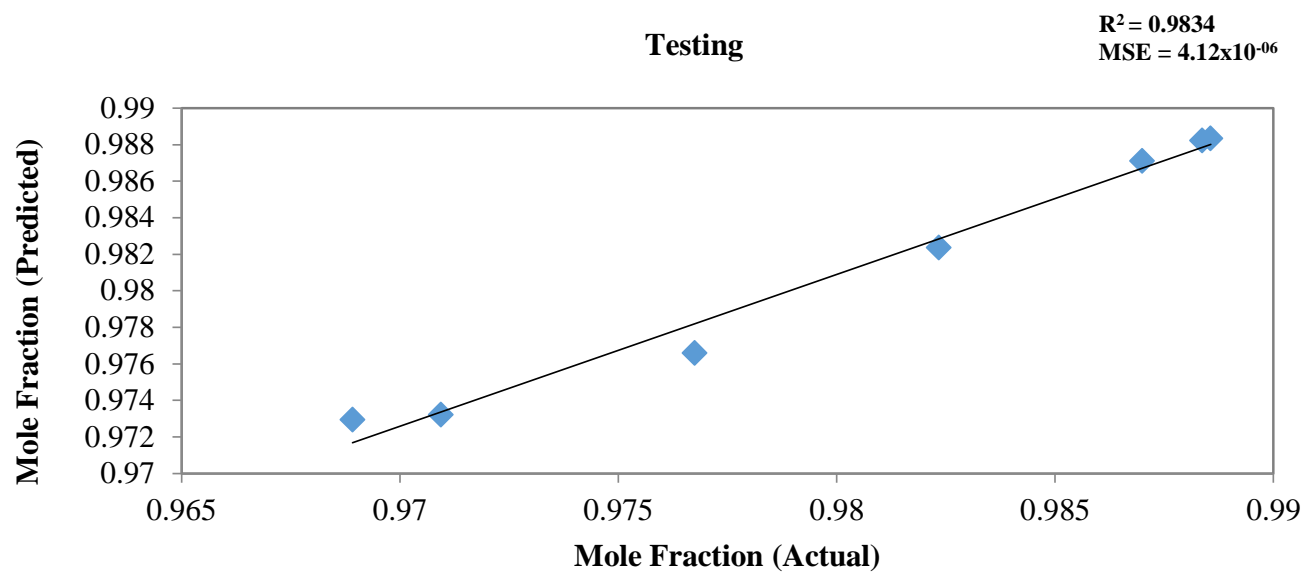

Figure 3 Testing curve of network

\section{Conclusion and future work}

Soft sensor was developed for the estimation of nbutyl acetate mole fraction in bottoms product of a reactive distillation column. The input data for the soft sensor was obtained from MATLAB model of RDC. A 3-layered ANN network was developed using Levenberg-Marquardt optimization technique. It has been observed that ANN based soft sensor worked perfect as an inferential estimator giving predictions with low mean square error and high $\mathrm{R}^{2}$ value.

\section{Acknowledgment}

None.

\section{Conflicts of interest}

The authors have no conflicts of interest to declare.

\section{References}

[1] Zamprogna E, Barolo M, Seborg DE. Composition estimations in a middle-vessel batch distillation column using artificial neural networks. Chemical Engineering Research and Design. 2001; 79(6):68996.

[2] Zamprogna E, Barolo M, Seborg DE. Estimating product composition profiles in batch distillation via partial least squares regression. Control Engineering Practice. 2004; 12(7):917-29.

[3] Zamprogna E, Barolo M, Seborg DE. Optimal selection of soft sensor inputs for batch distillation columns using principal component analysis. Journal of Process Control. 2005; 15(1):39-52.

[4] Venkateswarlu C, Kumar BJ. Composition estimation of multicomponent reactive batch distillation with optimal sensor configuration. Chemical Engineering Science. 2006; 61(17):5560-74.
[5] Qiao J, Fang Z, Chai T. LS-SVR-Based soft sensor model for cement clinker calcination process. In 2010 international conference on measuring technology and mechatronics automation 2010 (pp. 591-4). IEEE.

[6] De Canete JF, del Saz-Orozco P, Gonzalez S, GarcíaMoral I. Dual composition control and soft estimation for a pilot distillation column using a neurogenetic design. Computers \& Chemical Engineering. 2012; 40:157-70.

[7] Hong SJ, Jung JH, Han C. A design methodology of a soft sensor based on local models. Computers \& Chemical Engineering. 1999; 23:S351-4.

[8] Park S, Han C. A nonlinear soft sensor based on multivariate smoothing procedure for quality estimation in distillation columns. Computers \& Chemical Engineering. 2000; 24(2):871-7.

[9] Dong D, McAvoy TJ, Chang L. Emission monitoring using multivariate soft sensors. In proceedings of the American control conference, 1995 (pp. 761-5). IEEE.

[10] Wang X, Luo R, Shao H. Designing a soft sensor for a distillation column with the fuzzy distributed radial basis function neural network. In decision and control 1996. Proceedings of the 35th IEEE conference on 1996 (pp. 1714-19). IEEE.

[11] Fortuna L, Graziani S, Xibilia MG. Soft sensors for product quality monitoring in debutanizer distillation columns. Control Engineering Practice. 2005; 13(4):499-508.

[12] Bahar A, Özgen C. State estimation and inferential control for a reactive batch distillation column. Engineering Applications of Artificial Intelligence. 2010; 23(2):262-70.

[13] Khazraee SM, Jahanmiri AH. Composition estimation of reactive batch distillation by using adaptive neurofuzzy inference system. Chinese Journal of Chemical Engineering. 2010; 18(4):703-10.

[14] Rogina A, Šiško I, Mohler I, Ujević Ž, Bolf N. Soft sensor for continuous product quality estimation (in crude distillation unit). Chemical Engineering Research and Design. 2011; 89(10):2070-7. 
[15] Raghavan SV, Radhakrishnan TK, Srinivasan K. Soft sensor based composition estimation and controller design for an ideal reactive distillation column. ISA Transactions. 2011; 50(1):61-70.

[16] Jain P, Rahman I, Kulkarni BD. Development of a soft sensor for a batch distillation column using support vector regression techniques. Chemical Engineering Research and Design. 2007; 85(2):283-7.

[17] Gholami AR, Shahbazian M. Soft sensor design based on fuzzy c-means and RFN_SVR for a stripper column. Journal of Natural Gas Science and Engineering. 2015; 25:23-9.

[18] Behnasr M, Jazayeri-Rad H. Robust data-driven soft sensor based on iteratively weighted least squares support vector regression optimized by the cuckoo optimization algorithm. Journal of Natural Gas Science and Engineering. 2015; 22:35-41.

[19] Olanrewaju MJ, Al-Arfaj MA. Estimator-based control of reactive distillation system: Application of an extended Kalman filtering. Chemical Engineering Science. 2006; 61(10):3386-99.

[20] Sharma N, Singh K. Model predictive control and neural network predictive control of TAME reactive distillation column. Chemical Engineering and Processing: Process Intensification. 2012; 59:9-21.

[21] Dohare RK, Singh K, Kumar R. Modeling and model predictive control of dividing wall column for separation of Benzene-Toluene-o-Xylene. Systems Science \& Control Engineering. 2015; 3(1):142-53.

[22] Sharma N, Singh K. Neural network and support vector machine predictive control of tert-amyl methyl ether reactive distillation column. Systems Science \& Control Engineering. 2014; 2(1):512-26.

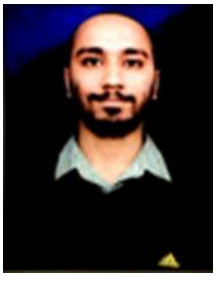

Gaurav Kataria is a $\mathrm{Ph} . \mathrm{D}$. research scholar in Department of Chemical Eengineering in MNIT, Jaipur. He received his B.Tech degree in Chemical Engineering from NIT Jalandhar and M.Tech degree in Chemical Engineering from MNIT Jaipur. Email:gkataria64@gmail.com

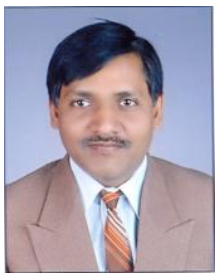

Dr. Kailash Singh has a B.E from University of Roorkee in 1992, M.Tech from IIT Kanpur, India in 1997 and Ph.D from Curtin University of Technology, Australia in 2007. Now he is working as Associate Professor in the Department of Chemical Engineering at Malaviya National Institute of Technology Jaipur. His Research areas are modelling and simulation and advanced pocess control.

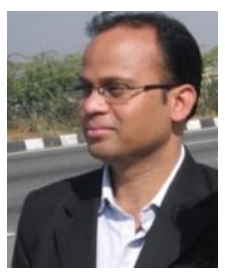

Dr. Rajeev Kumar Dohare has a B.E from NIT Surat in 2002, M.Tech from Aligarh Muslim University, Aligarh, and Ph.D from MNIT Jaipur, India in 2014. Now he is working as Assistant Professor in the Department of Chemical Engineering at Malaviya National Institute of Technology Jaipur. His Research areas are process modeling, simulation and control, solid waste management, fluid mechanics, and processes optimization. 